\title{
TECHNOLOGICAL INHERITANCE IN METAL FORMING
}

After various modes of metal forming by pressure, technological heredity manifests itself residual stresses, hardening, deformation gradient, residual plasticity and other factors that affect the performance properties of products.

It is necessary to create a methodology to quantify them.

Most of the indicators of technological heredity have already been studied [1], however, some, for example, the plasticity of a pre-deformed workpiece is not enough.

The purpose of this study is to develop a method for assessing the plasticity anisotropy of a pre-deformed metal under various types of stress state.

The measure of plasticity at the moment of destruction of the workpiece material in the region of finite deformations was taken to be the intensity of accumulated deformation (Udqvist parameter) [2], called the limiting:

$$
e_{p}=\int_{0}^{\tau_{p}} \dot{\varepsilon}_{u} d \tau
$$

where $\dot{\varepsilon}_{u}-$ strain rate intensity.

The plasticity of metals is determined by temperature, deformation rate, type of stress state, history of deformation, deformation gradient and other factors.

The dependence of plasticity on the type of stress state under simple deformation and fixed temperature-rate conditions is called the plasticity diagram.

To construct a plasticity diagram, a material is tested under various stress states under simple loading conditions, when the stress tensor components vary in proportion to one parameter.

It is customary to characterize the tense state by its indicators. In particular, according to the data of G. A. Smirnov-Alyaev [3], this indicator is assumed to be:

$$
\eta=\frac{I_{1}\left(T_{\sigma}\right)}{\sqrt{3 I_{2}\left(D_{\sigma}\right)}}=\frac{\sigma_{1}+\sigma_{2}+\sigma_{3}}{\sigma_{1}}
$$

where $I_{1}\left(T_{\sigma}\right)$ - the first invariant of the stress tensor;

$\sigma_{1}, \sigma_{2}, \sigma_{3}$-are the principal stresses;

$I_{2}\left(D_{\sigma}\right)$ - the second invariant of the stress deviator or stress intensity:

$$
\sigma_{u}=\frac{1}{\sqrt{2}} \sqrt{\left(\sigma_{1}-\sigma_{2}\right)^{2}+\left(\sigma_{2}-\sigma_{3}\right)^{2}+\left(\sigma_{1}-\sigma_{3}\right)^{2}}
$$

The parameter $\eta$ is used to construct plasticity diagrams in coordinates $e_{p}=f(\eta)$. Under uniaxial tension and compression $\eta=1$ and $\eta=-1$, respectively. For biaxial $\eta=2$ and $\eta=-2$, respectively, and for a shift $\eta=0$.

This indicator, reflecting the influence of the third invariant of the tensor or stress deviator, according to V. A. Ogorodnikov [4]:

$$
\chi=\frac{\sqrt[3]{I_{3}\left(T_{\sigma}\right)}}{\sqrt{3 I_{2}\left(D_{\sigma}\right)}}=\frac{\sqrt[3]{\sigma_{1} \sigma_{3} \sigma_{3}}}{\sigma_{u}}
$$


or

$$
\chi=\frac{\sqrt[3]{I_{3}\left(D_{\sigma}\right)}}{\sqrt{3 I_{2}\left(D_{\sigma}\right)}}=\frac{\sqrt[3]{S_{1} S_{3} S_{3}}}{\sigma_{u}}
$$

where $S_{1}=\sigma_{1}-\sigma$;

$S_{2}=\sigma_{2}-\sigma$;

$S_{3}=\sigma_{3}-\sigma-$ principal deviators of the stress tensor;

$\sigma_{u}=\frac{\sigma_{1}+\sigma_{2}+\sigma_{3}}{3}-$ medium strain.

They are often used to assess the used plastic resource of workpieces deformed under conditions of volumetric stress state.

The plasticity diagrams mentioned above can be represented by two-dimensional $e_{p}(\eta)$ or three-dimensional $e_{p}(\eta, \chi)$ diagrams.

When processing sheet materials by pressure, fracture can be accompanied by tearing or shearing, and sometimes mixed types of destruction. Therefore, we offer other indicators of the stress state.

When modeling fracture by separation, we assume that at constant strain rates and temperature, the plasticity diagram has the form of a dependence that is uniform for different stress states $e_{p}(\beta)[5]$, where:

$$
\begin{gathered}
\beta=\frac{1-S \eta}{\gamma} \\
\gamma=\frac{\sigma_{1}}{\sigma_{u}} .
\end{gathered}
$$

Here $\sigma_{1}$ is the largest of the principal stresses;

$S$ - material parameter.

When destroyed by a shearing, the diagram is described by the function $e_{p}(\theta)$, where:

$$
\begin{gathered}
\theta=\frac{1-k \eta}{\omega} \\
\omega=\frac{\tau_{\max }}{\sigma_{u}} .
\end{gathered}
$$

Here $\tau_{\max }$ - maximum shear stress; $k$ - material parameter.

Thus, the diagrams of plasticity $e_{p}(\eta, \chi, \theta, \beta)$ describe the change in the limiting deformation depending on the stress state indicators. They are obtained by testing materials under conditions of linear or plane stress states (tension, compression, torsion (shear)).

It is necessary to find out whether it is possible to construct such a diagram after preliminary plastic deformation at any stress state?

The solution to this problem is based on the tensor description of damage accumulation [6].

If the initial plasticity diagram, components of the damage tensor $\psi_{x}, \psi_{x y}, \ldots$ and the accumulated deformation at the end of plastic deformation, defined in the $\mathrm{X}, \mathrm{Y}, \mathrm{Z}$ coordinate system are known, then the plasticity diagram of the deformed metal can be constructed. 


\section{RESULTS AND ITS DISCUSSION}

Let it be required to determine the plasticity of a material $e_{p}^{\prime}$ under a stress state, which corresponds to its indicators $\eta_{1}^{\prime}, \eta_{2}^{\prime}$ and tensors $\beta_{x}^{\prime}, \beta_{x y}^{\prime}, \ldots$.

Then the increments of the components of the damage tensor upon additional deformation before fracture

$$
\begin{array}{r}
\Delta \psi_{x}=\beta_{x}^{\prime}\left[\varphi\left(e_{i}^{*}+e_{p}, \eta_{1}^{\prime}, \eta_{2}^{\prime}\right)-\varphi\left(e_{i}^{*}, \eta_{1}^{\prime}, \eta_{2}^{\prime}\right)\right] \\
\Delta \psi_{x}=\beta_{x y}^{\prime}\left[\varphi\left(e_{i}^{*}+e_{p}, \eta_{1}^{\prime}, \eta_{2}^{\prime}\right)-\varphi\left(e_{i}^{*}, \eta_{1}^{\prime}, \eta_{2}^{\prime}\right)\right]
\end{array}
$$

We write the destruction condition in the form

$$
\left(\psi_{x}+\Delta \psi_{x}\right)^{2}+\left(\psi_{x y}+\Delta \psi_{x y}\right)^{2}+\left(\psi_{y x}+\Delta \psi_{y x}\right)^{2}+\ldots=1
$$

or

$$
\Delta \psi_{x}^{2}+\Delta \psi_{x y}^{2}+\Delta \psi_{y x}^{2}+\ldots+2\left(\psi_{x} \Delta \psi_{x}+\psi_{x y} \Delta \psi_{x y}+\psi_{y x}+\ldots\right)+\psi_{0}^{2}=1
$$

where

$$
\psi_{0}^{2}=\psi_{x}^{2}+\psi_{x y}^{2}+\psi_{y x}^{2}+\ldots
$$

From equalities (1), (3) we obtain a quadratic equation, from which we find

$$
\varphi\left(e_{i}^{*}+e_{p}, \eta_{1}^{\prime}, \eta_{2}^{\prime}\right)=\varphi\left(e_{i}^{*}, \eta_{1}^{\prime}, \eta_{2}^{\prime}\right)-D+\sqrt{1+D^{2}-\psi_{0}^{2}}
$$

where

$$
D=\beta_{x}^{\prime} \psi_{x}+\beta_{x y}^{\prime} \psi_{x y}+\beta_{y x}^{\prime} \psi_{y x}+\ldots
$$

After approximation, the plasticity of the deformed metal

$$
e_{p}^{\prime}=e_{p}\left[-\frac{e_{i}^{*}}{e_{p}}-\frac{1-a}{2 a}+\sqrt{\left(\frac{e_{i}^{*}}{e_{p}}+\frac{1-a}{2 a}\right)^{2}-\frac{D}{a}+\frac{1}{a} \sqrt{1+a^{2}-\psi_{0}^{2}}}\right]
$$

Here $e_{p}$ - plasticity of non-deformed metal under stress state with indicators

$$
\eta_{1}=\eta_{1}^{\prime} ; \eta_{2}=\eta_{2}^{\prime} ;
$$

$a$-approximation coefficient [6]:

$$
\psi_{i j}=\int_{0}^{e_{u}}\left(1-a+2 a e_{u}^{*} / e_{p}\right) \beta_{i j} d e_{u}^{*} / e_{p} .
$$

According to known experimental data [6] $a=0.5$. Thus, according to equation (4), it is possible to calculate the ultimate deformation of the metal at a given indicator of the stress state.

Using the phenomenological theory of deformability, which describes the accumulation of damage by tensor models, it is possible to predict the technological heritage of the material as the residual plasticity of the resulting part. 
If during processing the components of the damage tensor at a given point of the workpiece are $\mathrm{C}$, then during the subsequent tensile test in the direction of the main axis at this point, the tensor components change by the value $\Delta \psi_{\mathrm{ij}}$.

If the fracture condition is written in the form (2), then the ultimate additional tensile deformation can be determined [6]

$$
\frac{e_{p 11}}{e_{p}}=-\frac{1}{2}-\frac{e_{i}^{*}}{e_{p}}+\sqrt{\left(\frac{1}{2}+\frac{e_{i}^{*}}{e_{p}}\right)^{2}-\sqrt{6} \psi_{11}+\sqrt{6 \psi_{11}^{2}+4\left(1-\psi_{i j} \psi_{j i}\right.}}
$$

where $e_{i}^{*}$ - accumulated deformation during the shaping of the workpiece;

$e_{p}=e_{p}(\eta=1)-$ plasticity of metal at $\eta=1$;

$e_{p 11}$ - residual plasticity in tension in the direction of the highest normal stress.

Since the parameter $\psi_{11}$ depends on the main direction $\sigma_{1}$, the residual plasticity $e_{p 11}$ also depends on it. Thus, using formula (5), one can estimate the anisotropy of plasticity during cold plastic deformation.

The practical significance of the results is evidenced by the assessment of the plasticity of steeply bent elbows obtained by the method of cold plastic deformation, according to a combined scheme, which includes deforming pulling of the unwilling workpiece. In this case, the workpiece in the form of a pipe was subjected to plastic bending. Earlier, in [7], a formula was obtained with the help of which it is possible to estimate the residual plasticity of the finished bend:

$$
\begin{aligned}
& \frac{e_{p}}{\delta_{p}}=-\frac{D_{1} b}{200}-\frac{e_{i}^{*}}{\delta_{p}}+ \\
& +\frac{D_{1} b}{100} \sqrt{\left(\frac{1}{2}+\frac{100 e_{i}^{*}}{D_{1} b \delta_{p}}\right)^{2}+\sqrt{2}\left[\psi_{S}-\psi_{\theta}+\sqrt{\left(\psi_{S}-\psi_{\theta}\right)^{2}+\left(1-\psi_{i j} \psi_{j i}\right)}\right]},
\end{aligned}
$$

where $\delta_{\mathrm{P}}$ - relative elongation at break;

$b=\frac{C_{1}}{B_{1}}\left(C_{1}=1.03, B_{1}=0.55\right.$ for Steel 20; $C_{1}=1.08, B_{1}=0.67$ for steel X10CrNiTi18-9);

$D_{1}=0.66$ for steel 20 and 0.73 - for steel X10CrNiTi18-9;

$e_{i}^{*}$ - the accumulated intensity of deformations during the shaping of the bend;

$e_{p}$ - residual plasticity;

$\psi_{S}, \psi_{\theta}, \psi_{i j}, \psi_{j i}$ - components of the damage tensor, which can be determined following the known relations [7]:

$$
\begin{gathered}
\psi_{\alpha}=\psi_{\theta}=\frac{1}{4} \sqrt{\frac{2}{3}} \cdot\left[\frac{e_{i}}{e_{p(\eta=2)}}+\left(\frac{e_{i}}{e_{p(\eta=2)}}\right)^{2}\right] ; \\
\psi_{S}=-\left(\psi_{\alpha}-\psi_{\theta}\right)=-2 \psi_{\alpha} ; \\
\psi_{i j} \psi_{i j}=\frac{1}{4}\left[\frac{e_{i}}{e_{p(\eta=2)}}+\left(\frac{e_{i}}{e_{p(\eta=2)}}\right)^{2}\right]^{2} .
\end{gathered}
$$

Experimentally checked [7] the results of calculating the residual plasticity of workpieces, pre-deformed to obtain finished products. For this purpose, from the $90^{\circ}$ bends $57 \times 4$; $90^{\circ} 89 \times 4.5$, made of 20 steel $\left(\delta_{\mathrm{P}}=30 \%\right)$, flat specimens were cut from the outer zone for tensile testing in the longitudinal and circumferential directions of the steeply curved bend. It was found that their residual elongation is approximately the same (table 1). 
Table 1

Comparison of calculated and experimental values residual plasticity of workpiece metal

\begin{tabular}{|c|c|c|c|c|c|c|c|}
\hline \multirow{2}{*}{ Branch size } & \multicolumn{4}{|c|}{ Calculations } & \multicolumn{2}{c|}{ Experiment } & \multirow{2}{*}{ Max. } \\
\cline { 2 - 7 } & $\psi$ & $e_{P S}$ & $e_{P}$ & $e_{P \alpha}=e_{P \theta}$ & $e_{P \alpha}$ & $e_{P \theta}$ & divergence, \% \\
\hline $90^{\circ} 57 \times 4$ & 0,35 & 0,58 & 0,27 & 0,43 & 0,41 & 0,37 & 9,3 \\
\hline $90^{\circ} 89 \times 4,5$ & 0,40 & 0,59 & 0,25 & 0,41 & 0,38 & 0,35 & 14,6 \\
\hline
\end{tabular}

One of the most important indicators of the quality of bends obtained by the method of cold plastic deformation is their residual plasticity under operating loads (especially under cyclic pressure loads at elevated temperatures), which is estimated indirectly from the results of tensile tests of samples cut from bends in different directions.

\section{CONCLUSIONS}

A method is developed for determining the plasticity of a pre-deformed metal, based on a tensor description of damage accumulation under conditions of cold plastic deformation. It allows, when the mechanical characteristics are known, and also the plasticity diagrams are plotted, to evaluate the plasticity of pre-deformed blanks at any type of stress state. The method has been tested in the manufacture of steeply bent bends by pulling a pre-wired pipe. Revealed satisfactory convergence of the calculated and experimental data.

A method for estimating the plasticity of a pre-deformed metal is developed based on the tensor description of damage accumulation under conditions of cold plastic deformation. The method makes it possible, with known mechanical characteristics, and also with known plasticity diagrams, to evaluate the plasticity of pre-deformed blanks for any kind of the stress state. The method has been tested using the example of manufacturing steeply curved bends by means of pulling a presewn pipe. Satisfactory convergence of the calculated and experimental data is shown.

\section{REFERENCES}

1. Yasheritsyn P.I., Ryzhov E.V., Averchenkov V.I. Technological heredity in mechanical engineering. Minsk: Science and Technology. 1977. 256 p. (in Russian).

2. Kachanov LM Fundamentals of the theory of plasticity. Moscow: Science, 1969. 420 p. (in Russian).

3. Smirnov-Alyaev G.A. Resistance of materials to plastic deformation. Leningrad: Mechanical Engineering. 1978. 368 p. (in Russian).

4. Ogorodnikov V. A. Assessment of the deformability of metals during pressure processing. Kyiv: Higher School. 1983. 176 p. (in Russian).

5. Dell H., Gese H., Obezhover G.A. Comprehensive Approach for the Prediction of Sheet Metal Failure. Materials Proc. and Design, Modeling, Simulation and Applications. Part 1. Numiform 07. American Institute of Physics. 2007, pp. 165-170.

6. Del G.D. Plasticity of the deformed material. Physics and technology of high pressures. 1983. 11, pp. 28-32. (in Russian).

7. Rosenberg O.A., Ogorodnikov V.A., Grushko A.V., Studenets S.F., Melnichenko V.V. Shaping of steeply curved bends by the bending of a stretched pipe. Vinnytsia: Universum. 2004. 140 p. (in Russian).

Ogorodnikov Vitaliy A. - Doctor of Technical Sciences, Full Professor, VNTU; E-mail: va.ogorodnikov@gmail.com; https://orcid.org/0000-0002-0392-8897

Arkhipova Tetiana F. - PhD of Technical Sciences, Associate Professor, VNTU E-mail: tfarhipova@gmail.com; https://orcid.org/0000-0003-2279-1491

VNTU - Vinnitsa National Technical University, Vinnitsa. 